\title{
Providing A Secure Environment For E-Commerce Sites Using SSL Technology
}

\author{
Maha A. Sayal \\ Department of Computer Sciences, College of Computer Sciences and Math, Thi-Qar University, Iraq \\ Email: maha.asyal@utq.edu.iq
}

(Received May 03, 2018; Accepted October 03, 2018; Available online March 01, 2020)

DOI: 10.33899/edusj.2020.164371, (C) 2020, College of Education for Pure Science, University of Mosul.

This is an open access article under the CC BY 4.0 license (http://creativecommons.org/licenses/by/4.0/).

\begin{abstract}
:
The issue of security on the Internet is at the top of the interest for most users, especially those who want to buy online and therefore find a large majority of users, especially new ones refrain from buying online and are afraid to go into such an experiment to complete the image and know more safety in the use of credit cards for some websites.

In order to increase confidence in online business transactions, we have proposed a safe environment for commercial sites by installing a private server and then submitting on certificate authority for digital certificates and obtaining Secure Socket Layer SSL technology, certificate it, and adding it to the server as well as hosting sites to secure its data and renew the certificate at expiration. This allows the business website owner to host that website on the secure server so the customer information and data will be protected.
\end{abstract}

Keywords: commercial sites, Secure Socket Layer SSL, certificate authority, digital certificates, online business transactions

\section{توفير بيئة امنة لمواقع التجارة الالكترونية بأستعمال تقنية طبقة الفتحات الامنة (SSL)}

$$
\begin{aligned}
& \text { مها عبد اللطيف سايل } \\
& \text { قسم علوم الحاسبات, كلية علوم الحاسوب والرياضيات, جامعة ذي قار , العراق }
\end{aligned}
$$

ان ما يتصدر قائمة الاهتمامات لاى معظم مستعملي الانترنت هو موضوع الأمنية على شبكة الإنترنت وخصوصاً عندما

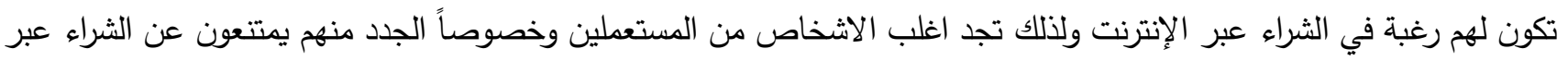
الإنترنت ويخشون من اعطاء معلومات عنهم حتى تكتمل الصورة لديهم و يتعرفون على مدى درجة الأمان في استعمال بطاقات الأئتمان في بعض المواقع.

ولزيادة الثقة بالصفقات والتعاملات التجارية عبر الانترنت اقترحنا توفير بيئة امنة للمواقع التجارية عن طريق تتصيب خادم

خاص ومن ثم التقديم على هيئة توثيق الثهادات الرقمية و الحصول على تقنية طبقة الفتحات الامنة Secure Socket Layer

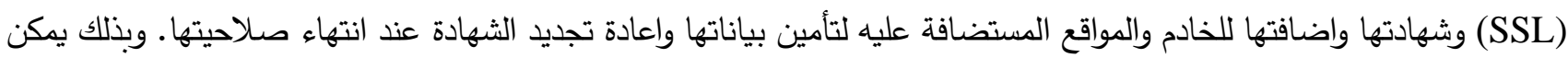
لصاحب الموقع التجاري ان يستضيف موقعه على ذلك الخادم الامن لحماية معطيات الزبون. 
الكلمات المفتاحية: المواقع التجارية, طبقة النتحات الامنة ,هيئة التوثيق,الثهادات الرقمية ,الصفقات التجارية عبر الانترنت .

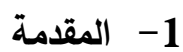

ان التجارة الاكترونية اصبحت جزءاً مهماً في بناء الاقتصاد مستقبلاً,كون التجارة هنا اصبحت متعلقة بالتكنولوجيا وما لها من

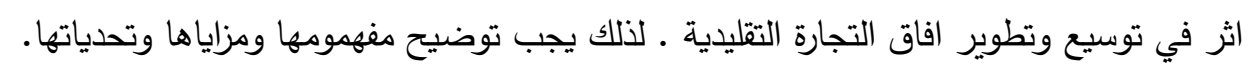

Electronic Commerce: 1-1 التجارة الالكترونية هي عملية شراء أو بيع أو تبادل المنتجات والمعلومات والخدمات من خلال الثبكات المحلية والدولية والعالمية ومن ضمنها الانترنت. فهي عملية تطبيق تقنية من أجل جعل المعاملات التجارية تجري بصورة تلقائية وسريعة هذا من وجه نظر الأبراء الأعمال الاقتصادية و التجارية [1]. ان التسوق في المجمعات التجارية الموجودة على الانترنت و بنوك الانترنت وشراء الاسهم والتعاون مع بقية الأفراد في عمل بحث ما هي من تطبيقات التجارة الالكترونية [2].و لتنفيذ هذه التطبيقات، يتطلب الحصول على دئ دعم المعلومات وبنية تحتية وأنظمة. تكون تطبيقات التجارة الالكترونية مدعمة بأساس وبننى تحتية .وتأدية عمل هذه التطبيقات يتطلب الاعتماد على أربعة اساسيات وهي

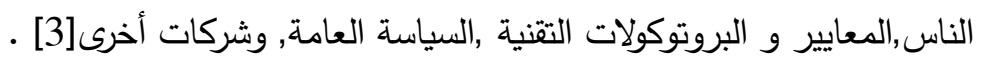
1-2-1 مزايا التجارة الاكترونية تتمتع التجارة الالكترونية بمجموعة من المزايا يمكن ايجازها :

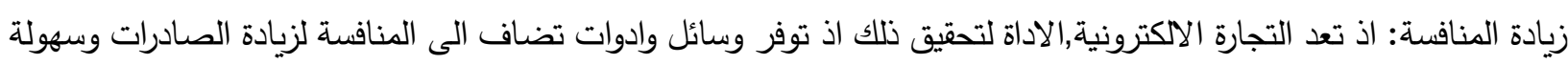
وصول المستهلكين الى البضائع من خلال مواقع الانترنت, ويمكن اجمال ميزاتها بما يلي: 1- امكانية التسويق على المستوى العالمي بكلفة محددة .

2- السرعة في عقد الصفقات وانهائها اذ يكون الاجتماع مع الشركات المتعاقدة على الانترنت بأستعمال برامج فيديوية واجراء

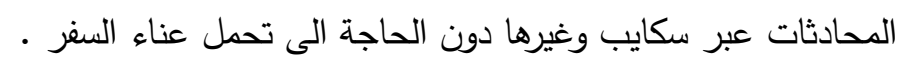

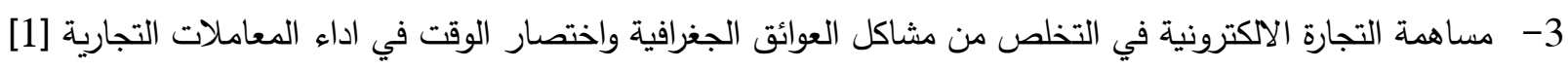
امكانية القيام بمشروعات صغيرة او متوسطة تعاني من غياب الموارد الاقتصادية اذ ان التجارة الاككترونية اعدت لكي تتمكن

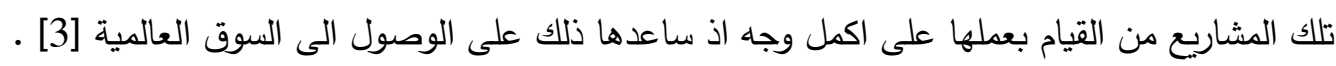

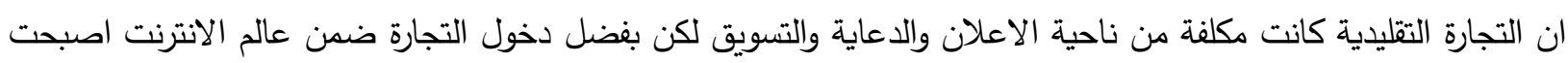

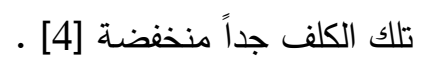
3-1

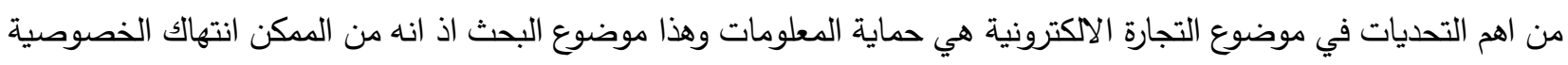

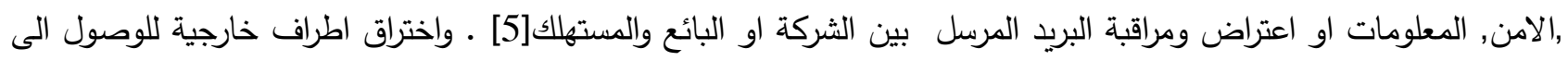
بيانات العملاء والتعرف على خصوصيات الثركة اذا انتفى وجود ساتر امني لان مجرد الوصول الى الحاسب الثخصي للعميل

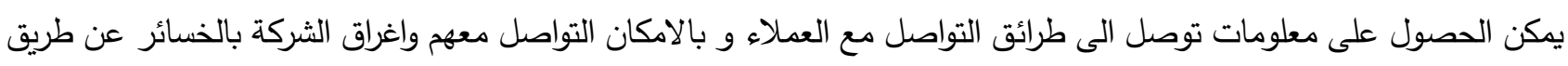


معرفة ميولهم ورغبات العملاء وبالتالي تحميل الشركة خسائر جمة وهذا سبب تعرض حسابات وبطاقات ائتمان العملاء والمشترين

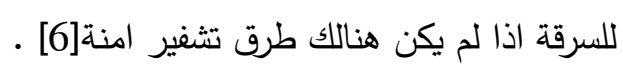
ومن هنا وجد انه لا بد من السعي لحل تلك المشكلة للأحتفاظ بالخصوصية والامان وحماية كلا من الثركة والعملاء والزبائن , اذ تكون حماية الخادم الخاص بالثركة التجارية من اجل الثركة والعميل وحماية الموقع التجاري من اجل الثركة والزبون اذ يتم تثفير المعلومات المارة بين الطرفين من خلال تقنية SSL واستعمالها في الثهادة الرقمية التي يمكن الحصول عليها من هيئة توثيق

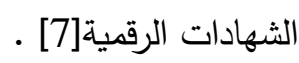

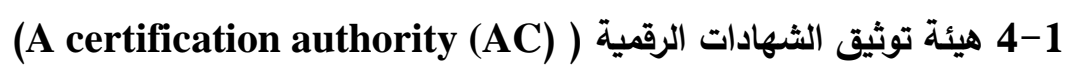

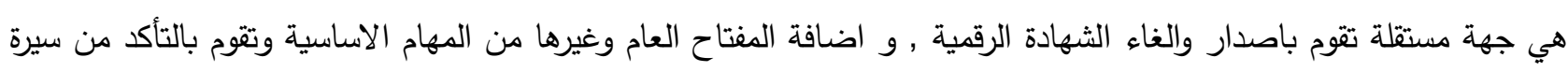

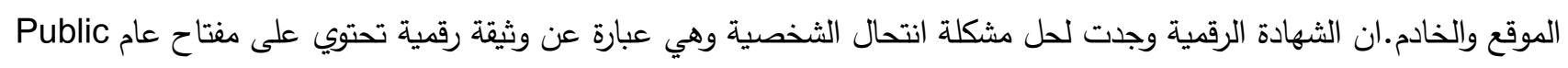

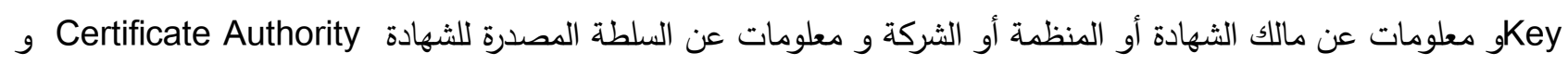

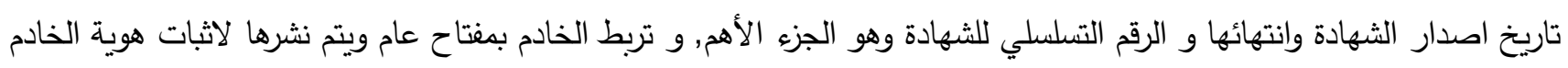

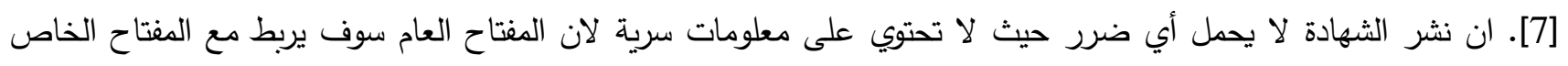
المعرف من قبل الكيان المالك للثهادة ـ واهمية الثهادة تكمن في اجراء المعاملات الرقمية ,عند الثراء عبر الانترنت وعند ارسال الرسائل لاثبات هوية الشخص.وكيفية معرفة الثخص بوجود شهادة رقمية للموقع , مثلا عند تحقق الثخص من حسابه البنكي عبر الثراه

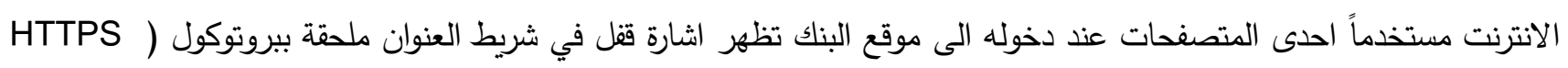
(Hypertext Transfer Protocol Secure وهو بروتوكول النصوص التشعبية الأمن وهذا يدل على ان الصفحة محمية ويستخدم شهادة رقمية لتأمين الصفحة , الثكل (1) [8] .

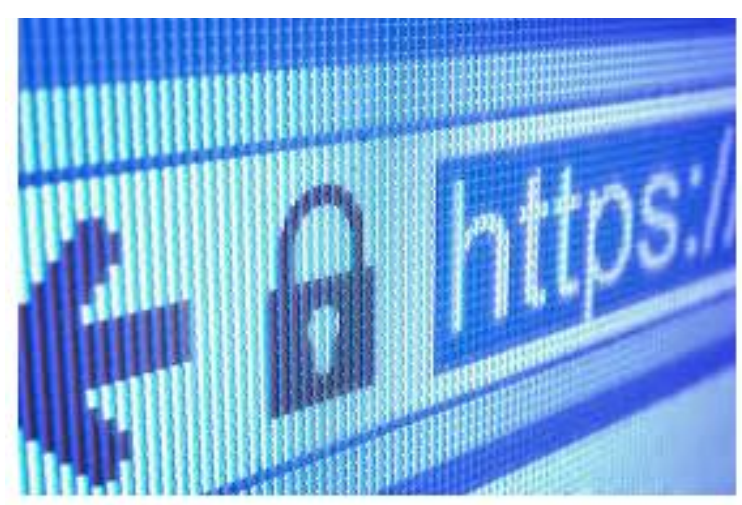

الثكل (1) يوضح وجود الثهادة الرقمية

1-5-1 تقنية طبقة الفتحات الامنة SSL وآلية عملها تقنية طبقة الفتحات الامنه SSL هي برنامج يحتوي على بروتوكول تثفير متخصص لنقل المعلومات والبيانات التي تم تثفيرها

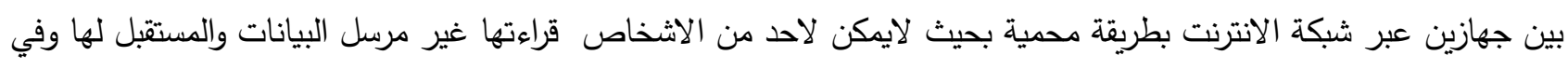
نفس الوقت تكون طريقة التشفير فيها معقدة ويصعب فكها, وهي تختلف عن بقية طرق التشفير في شى واحد الا وهو ان مرسل

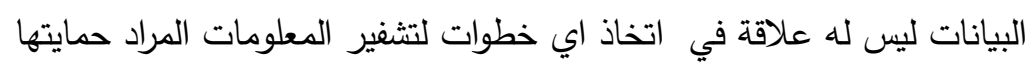

يقوم هذا البرنامج الذي يعمل بتلك التقنية بتشفير أي معلومة صادرة من ذلك المستعرض وصولاً إلى جهاز الخادم الخاص

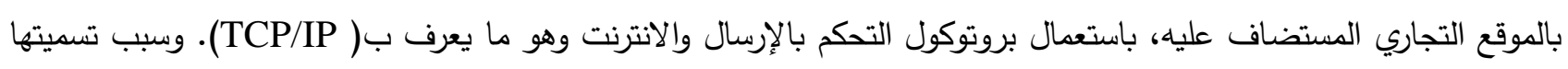


بالطبقة الامنة لأن هذا البرنامج يعمل كطبقة وسطية تربط بين بروتوكول التحكم بالنقل و بروتوكول نقل النصوص المتشعبة .[10] (HTTP)

$$
\text { وتقتخ خطوات استخدام هذه التقنية كالتالي [11] }
$$

1- تقوم هيئة التوثيق بإصدار الثهادة الرقمية الخاصة بالموقع بعد تقديم طلب من صاحب الموقع التجاري بحيث تحتوي على كل

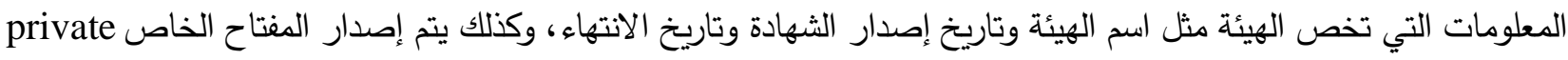
key و المفتاح العام public key ليتم تخزين SSL للموقع و يقوم الموقع أيضا بتأمين جهاز خادم مزود بيرنامج لتشفير المفتاح العام للموقع.

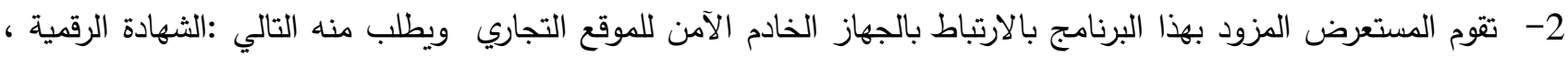
مصدرها ، تاريخ انتهاءها وكذلك تتم المقارنة بين اسم الموقع التجاري على الثهادة مع اسم الموقع في جهاز الخادم والمقارنة

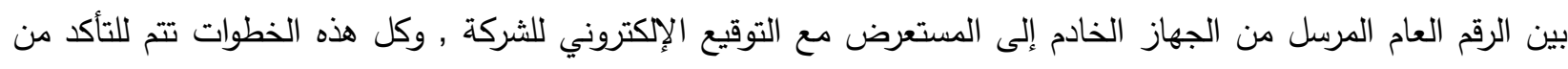
مصداقية الموقع وحماية الزبون من الثركات الوهية.

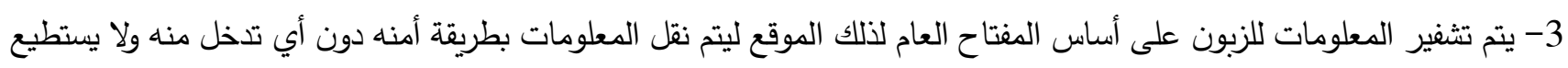
أحد سرقة المعلومات أو الإطلاع عليها سوى الموقع المعتمد في الطرف الآخر والذي يملك المفتاح الخاص للموقع لفتح وإعادة

$$
\text { 2- الجعانب العملي إلى وضعها الطبيعي • }
$$

ان ال SSL هو الطريق لتشفير معلومات الموقع وخلق ارتباط اكثر امان , بالإضافة الى ان شهادة ال SSL تعرض معلومات

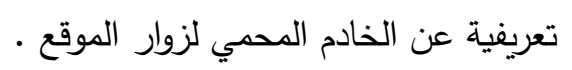

تم في هذا العمل اقتراح تنصيب خادم معين ومن ثم التقديم على هيئة توثيق الشهادات الرقمية و للحصول على تقنية SSL وشهادتها

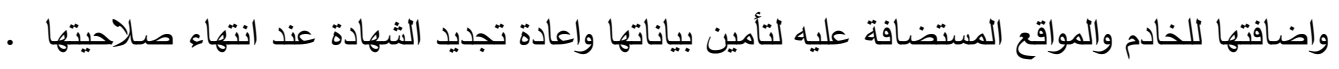
يوجز الثكل (2) مراحل توفير البيئة الامنة .

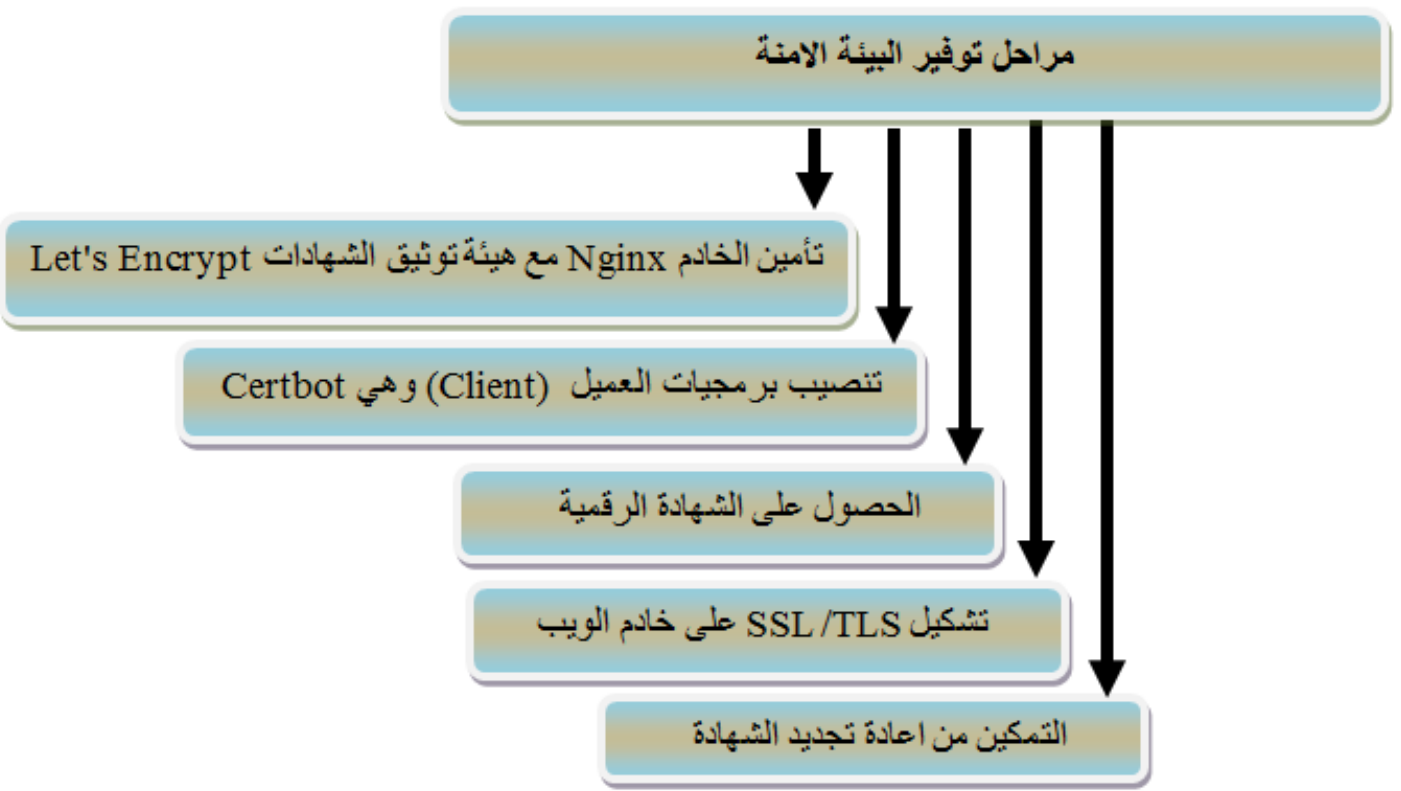

الثكل(2) ملخص مراحل توفير البيئة الامنة 


$$
\text { 1-2 البرمجيات ونظام التشغيل المستخدم في الجانب العملي }
$$

ان النظام المستخدم في العمل هو نظام Linux اصدار Ubuntu14.04 اذ ان مميزات هذا النظام انه مفتوح المصدر واكثر اماناً من نظام Windows لانه قابل للتحديث ملايين المرات في الدقيقة الواحدة , أي من الممكن اضافة خطوات لبرمجة جذر النظام

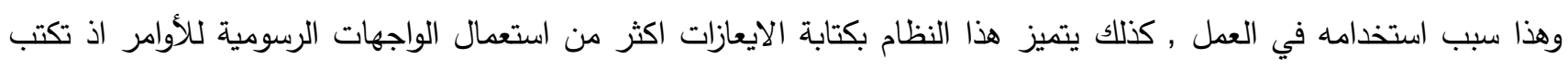

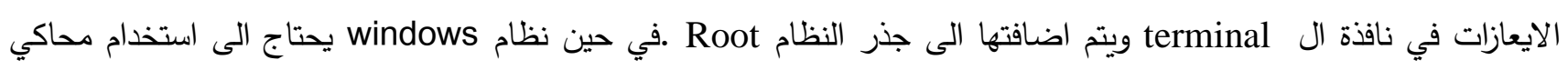
طرفي لأضافة بعض الاوامر المستعملة في البحث على النظام .

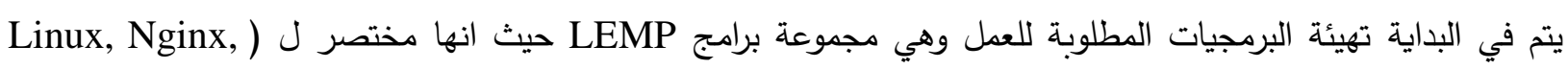

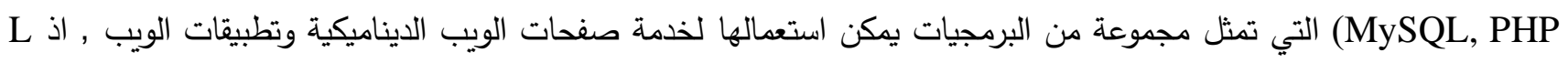

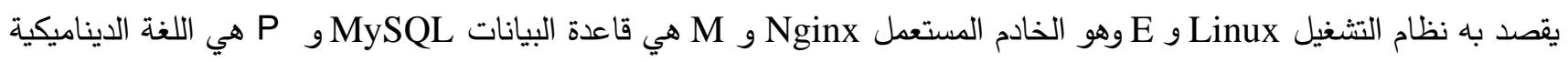

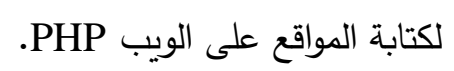
من الجدير بالذكر ان جميع الايعازات والبرمجة تكتب بنافذة (Terminal) الخاصة بنظام Sinux. Sudo apt-get update Sudo خطوات تتصيب الخادم Nginx تكون كالتالي : apt-get install nginx يوضح الثكل(3) خطوات تتصيب الخادم بصورة صحيحة و حجز مكان له على الثبكة العنكبوتية

Welcome, to nginxl - Mozilla Firefox
Welcome to nginx!
If you see this page, the nginx web server is successfully installed and working.
Further configuration is required.
For online documentation and support please refer to nginx.org.
Commercial support is available at nginx.com.
Thank you for using nginx.

\section{Nginx الشكل (3) تنصيب الخادم}

ملاحظة : في هذا البحث تعذر امكانية تعريب بعض الاشكال والجداول لاحتوائها على مصطلحات ومختصرات(معروفة علمياً). وفي تُصي

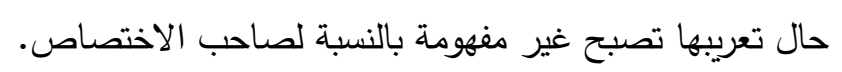

Let's Encrypt Nginx مع 2-2 تأمين الخادم

هي هيئة توثيق Let's Encrypt شهادات رقمية جديدة توفر اساليب سهلة للحصول على وتتصيب شهادات SSL و ومقدرتها على تخويل HTSP مشفر للعمل على خادمات الويب. الثكل(4) يوضح شعار هيئة التوثيق. 


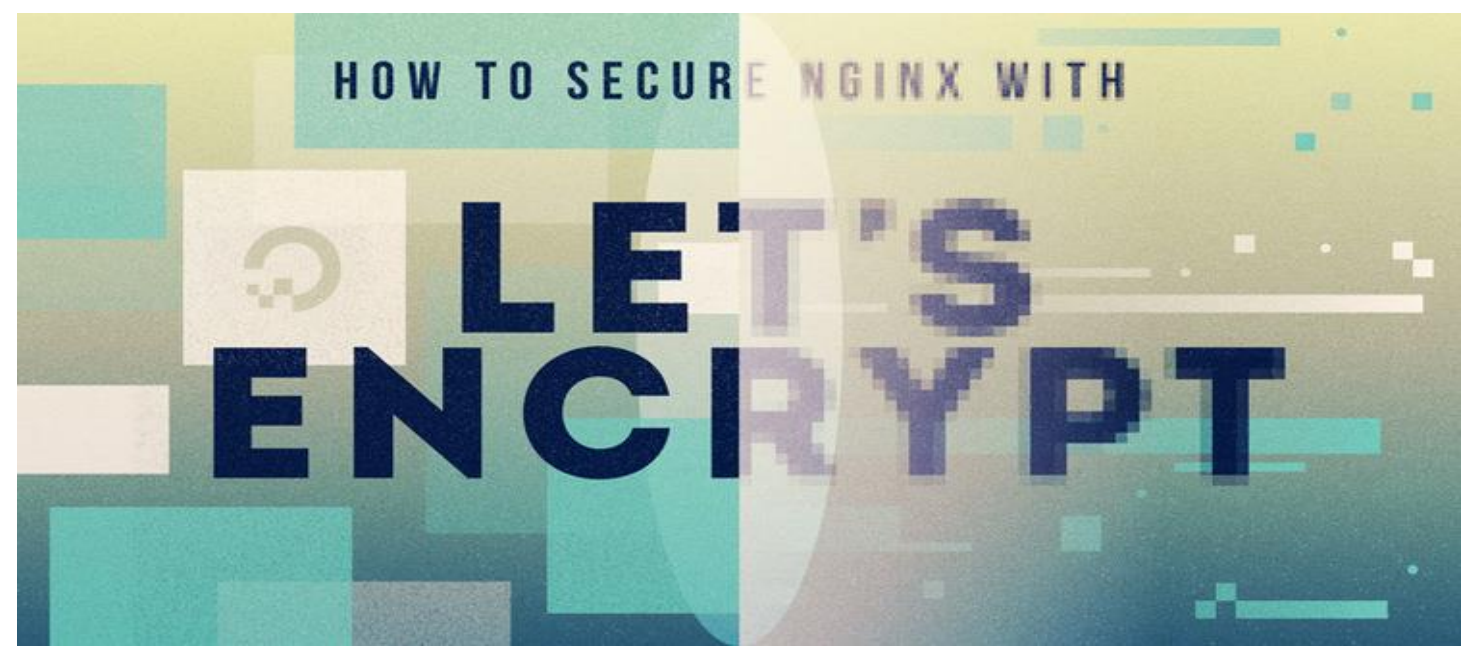

\section{الثكل (4) شعار هيئة التوثيق[12]}

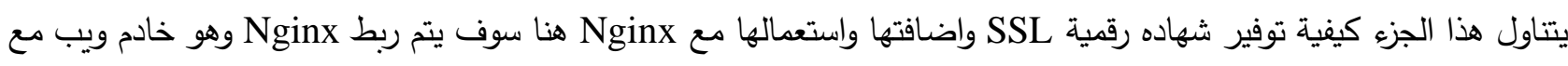

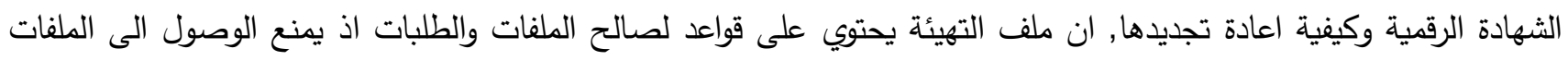
بالاسماء الموجودة في الملف xx /.well-known/acme-challenge/xxx.يين الثكل (5) الية منح الثهادات الرقمية.

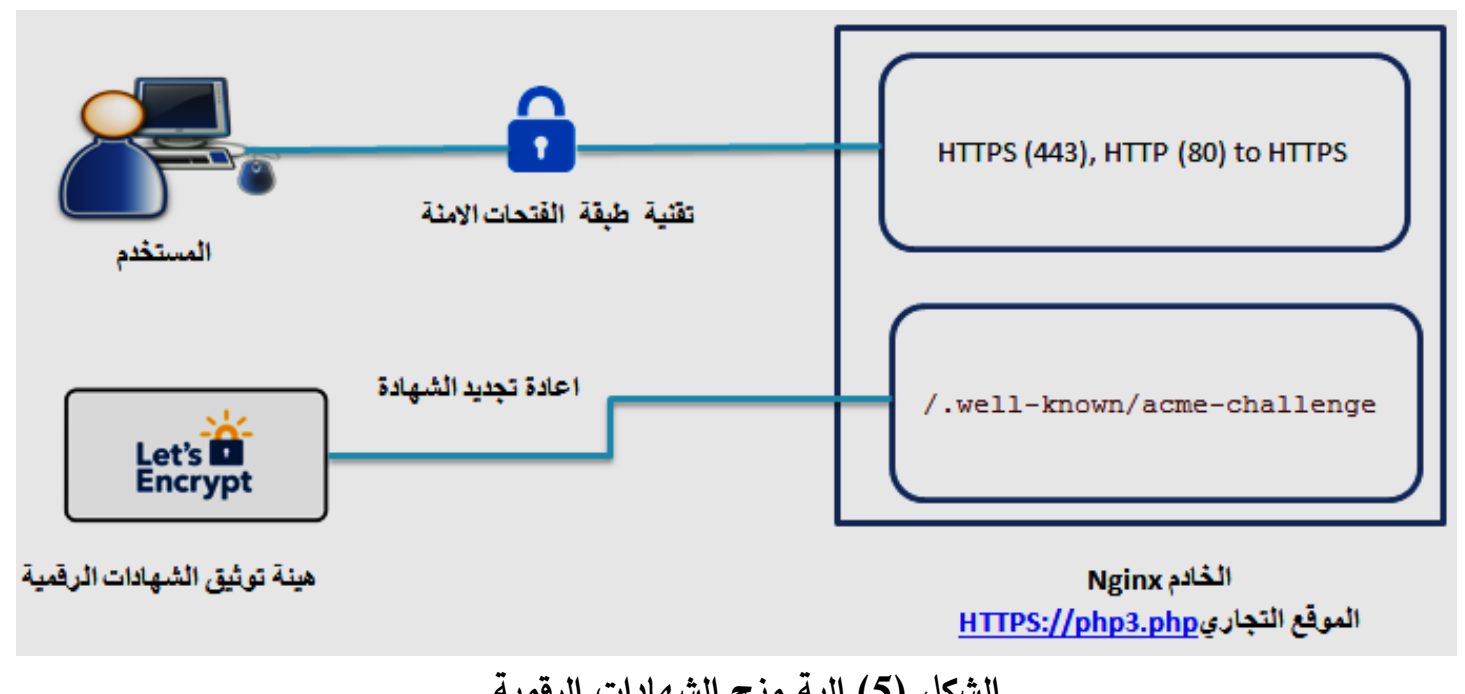

الشكل (5) الية منح الشهادات الرقمية

Certbot 3-2 تنصيب برمجيات العميل (Client) وهي (الخيل Certbot الحصول على شهادة SSL برمجياً بغض النظر عن خادم الويب المستعمل. في الخطوه الاولى يتم اضافة مستودع للتخزين من خلال الثفره التالية.

sudo add-apt-repository ppa:certbot/certbot

sudo apt-get update

sudo apt-get install certbot
في هذه الخطوه يتم بالتحديث واخيراً يتم بتصيب برمجيات العميل 


\section{2-4 الحصول على الثهادة الرقمية}

يوفر ال Let's Encrypt طرائق متنوعة للحصول على الثهادة , وهذه الطرائق او الاضافات تسمى مصادقة. اذ ان الاضافات تستعمل في حالة وجوب اصدار شهادة للخادم Nginx . ان الخطوة الاساسية الاولى في الحصول على الثهادة هي كيفية استعمال

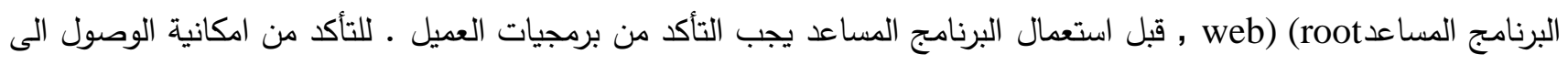

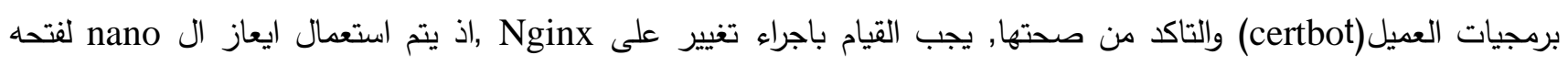

$$
\text { وتعديله }
$$

sudo nano /etc/nginx/sites-available/default وبعد تعديله يتم التحقق اذا كان فيه اخطاء من خلال تتفيذ الايعازات التالية :

sudo ngin $-\mathrm{t}$

sudo service nginx restart

certbot

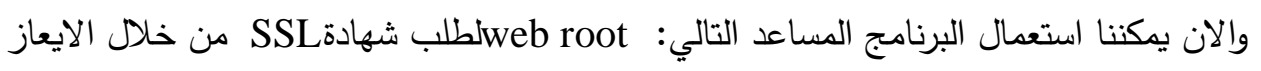
certonly --webroot --webroot-path=/usr/share/nginx/html -d example .com -d www.PHP3.com

هو اسم موقع تجاري مستضاف على الخادم, ومن ثم يتم اعطاء شهاده رقمية كما في الشكل(6) :

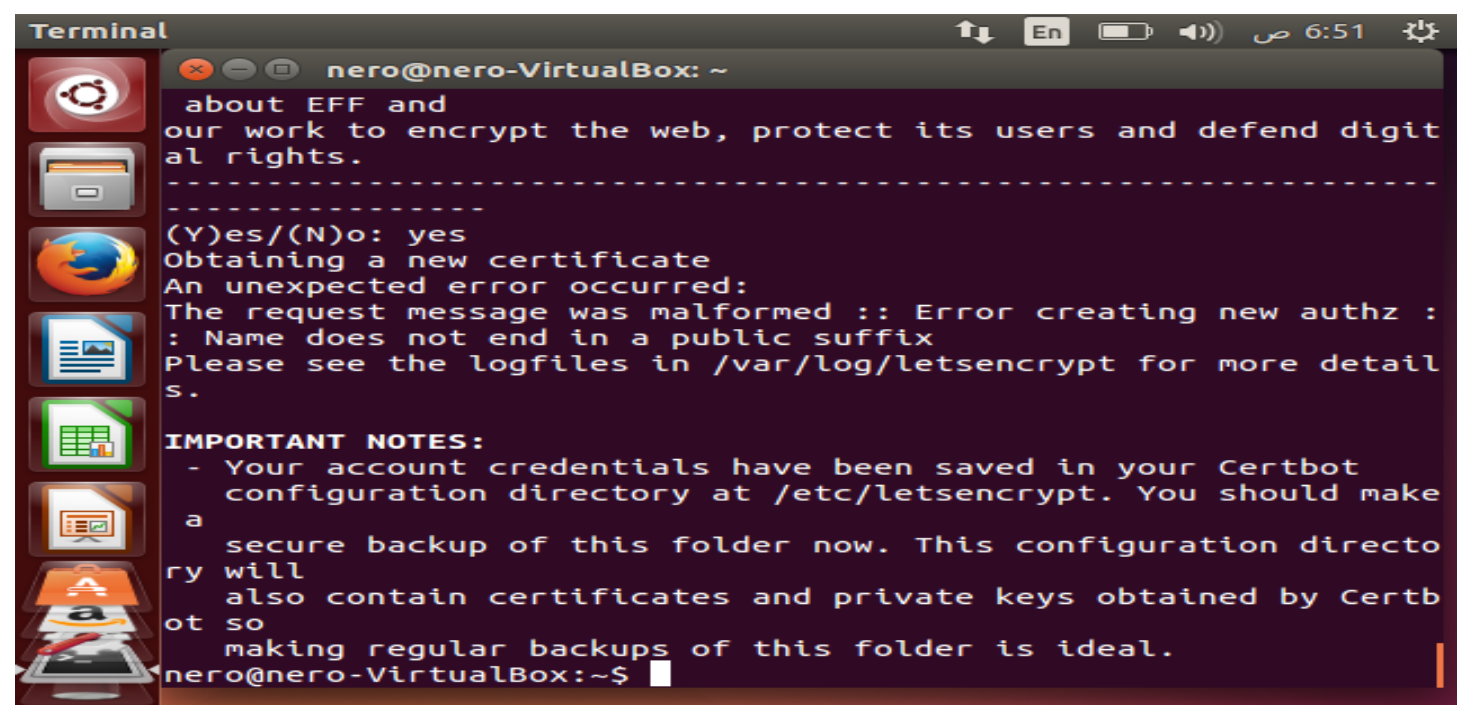

$$
\text { الشكل (6) منح الشهادة الرقمية للخادم }
$$

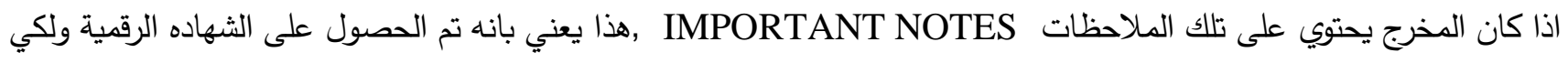

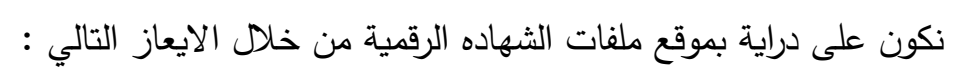
sudo ls -1/etc/letsencrypt/live/your_domain_name

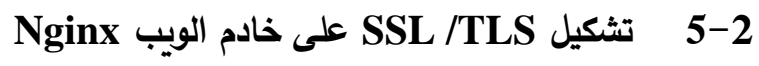
الان بعد ان تم امتلاك شهادة SSL , نحتاج الى تهيئة الخادم لأستعمالها لذلك نحرر بياناته الاولية لفتح ملف التهيئة للخادم

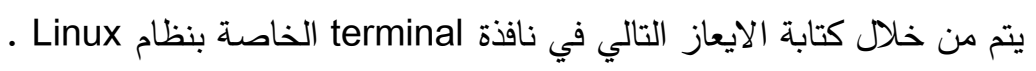


sudo nano /etc/nginx/sites-available/default

listen 80 default_server;

$$
\text { عند فتح الملف اولا يتم حذف الخطوتين }
$$

listen [::]:80 default_server ipv6only=on;

تم حذف المنفذ 80 الذي يستعمله الخادم افتراضياً, ثم تم اضافة المنفذ 443 مع اضافة امكانيات SSL

listen $443 \mathrm{ssl}$;

server_name example.com www.example.com;

ssl_certificate/etc/letsencrypt/live/example.com/fullchain.pem;

ssl_certificate_key/etc/letsencrypt/live/example.com/privkey.pem;

وهنا يتمكن الخادم من استعمال SSL واستعمال الثهادة التي حصلنا عليها من هيئة التوثيق بعدما نقوم باعادة تثغيل الخادم • sudo service Nginx restart 6-2 التمكين من اعادة تجديد الثهادة الالي في هذه المرحلة يتم اعطاء فتره زمنية للشهادة الرقمية وسوف تكون الثهادة الرقمية صالحة لمدة تسعين يوما هذا لتشجيع المستعملين على تجديد الثهادة الخاصه بهم سوف نحتاج الى اوامر تثغيل قانونية للتحقق من انتهاء صلاحية الشهادة وتجديدها

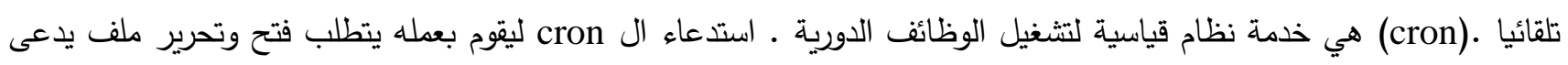
sudo crontab -e crontab نضيف النص التالي في نهاية الملف طن نالنعازن

$153 * *$ /usr/bin/certbot renew --quiet --renew-hook "/usr/sbin/service nginx reload" الرقم يعني تثغيل اوامر فحص التجديد في الساعة 3:15 am كل يوم ويمكن ان نختار أي وقت , لذلك يمكن تجديد الثهادة متى ما انتهت مدتها ـ هناك عدة اشكال توضح كيفية فتح الملف crontab والتعديل عليه.

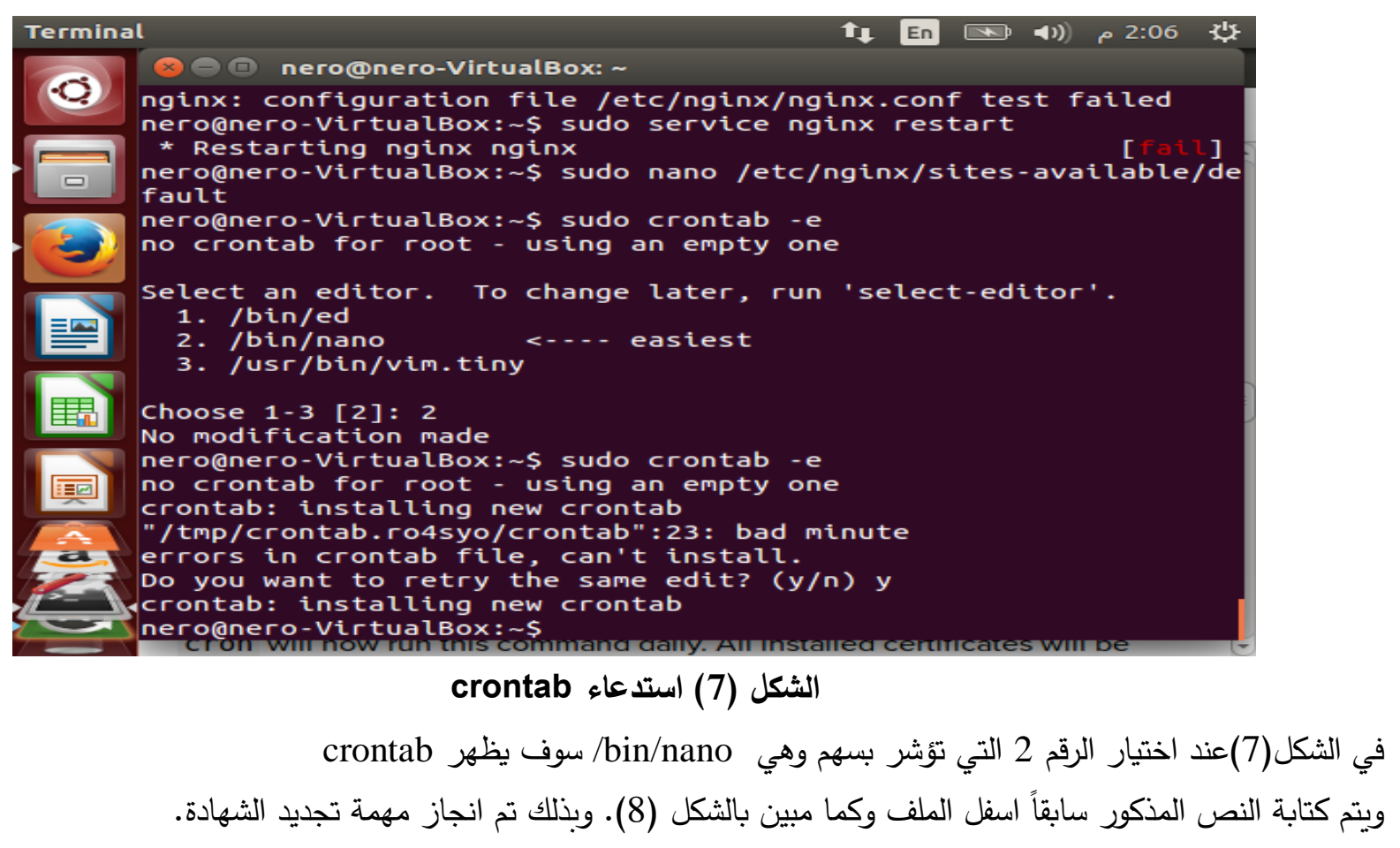




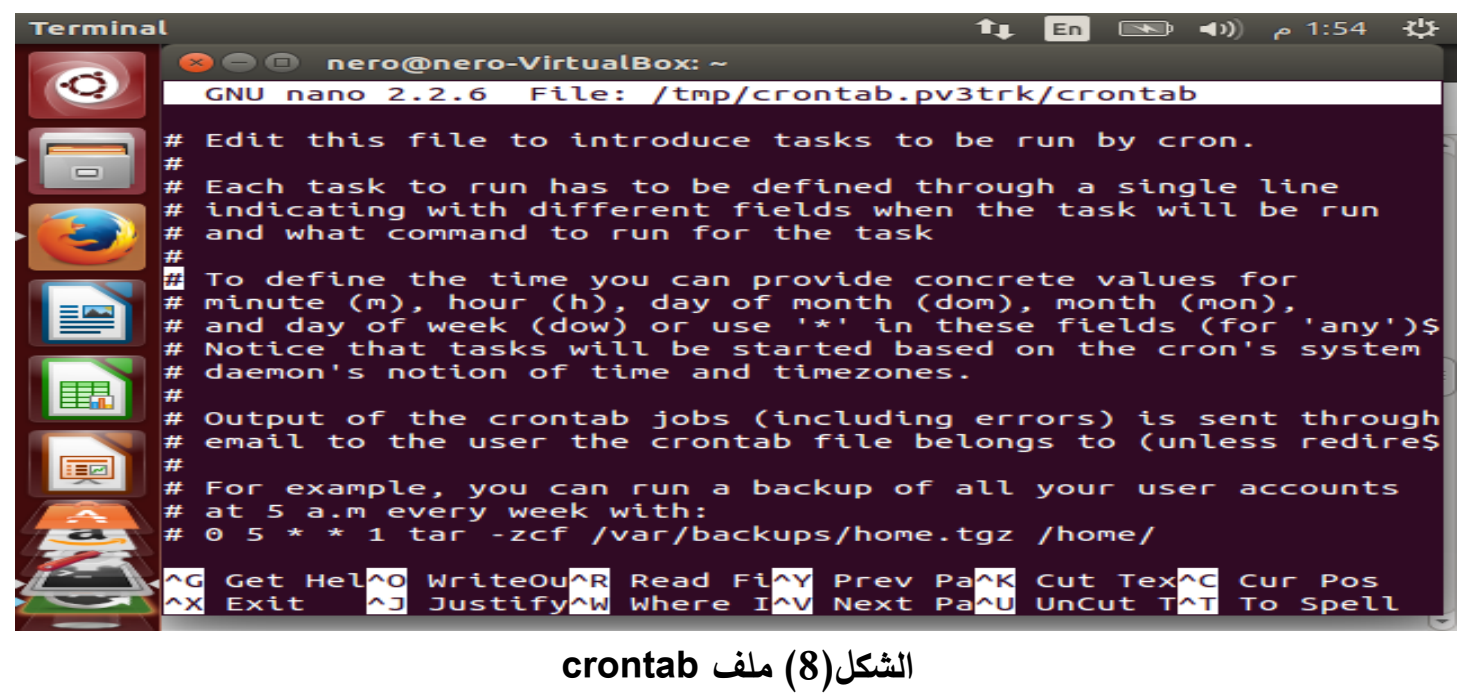

4-

تقنية و شهادة SSL الان طبقت من هيئة التوثيق عن طريق عمل موقع تجاري(php3.php)يضم مكتبة لبيع الكتب,

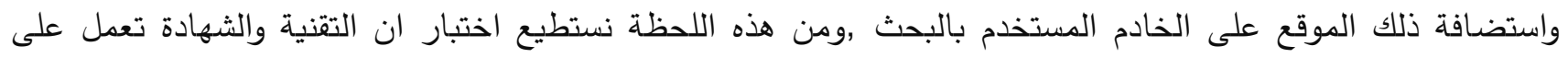
النحو الصحيح , وذلك عن طريق زيارة المجال الخاص بنا في موقع هيئة التوثيق عن طريق متصفح الويب من خلال

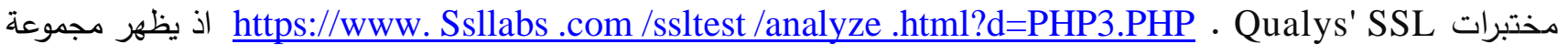
تقديرات اداء مواقع الخدمة على الخادم المستعمل والموقع المستضاف. وكما مبين بالثكل (9) •

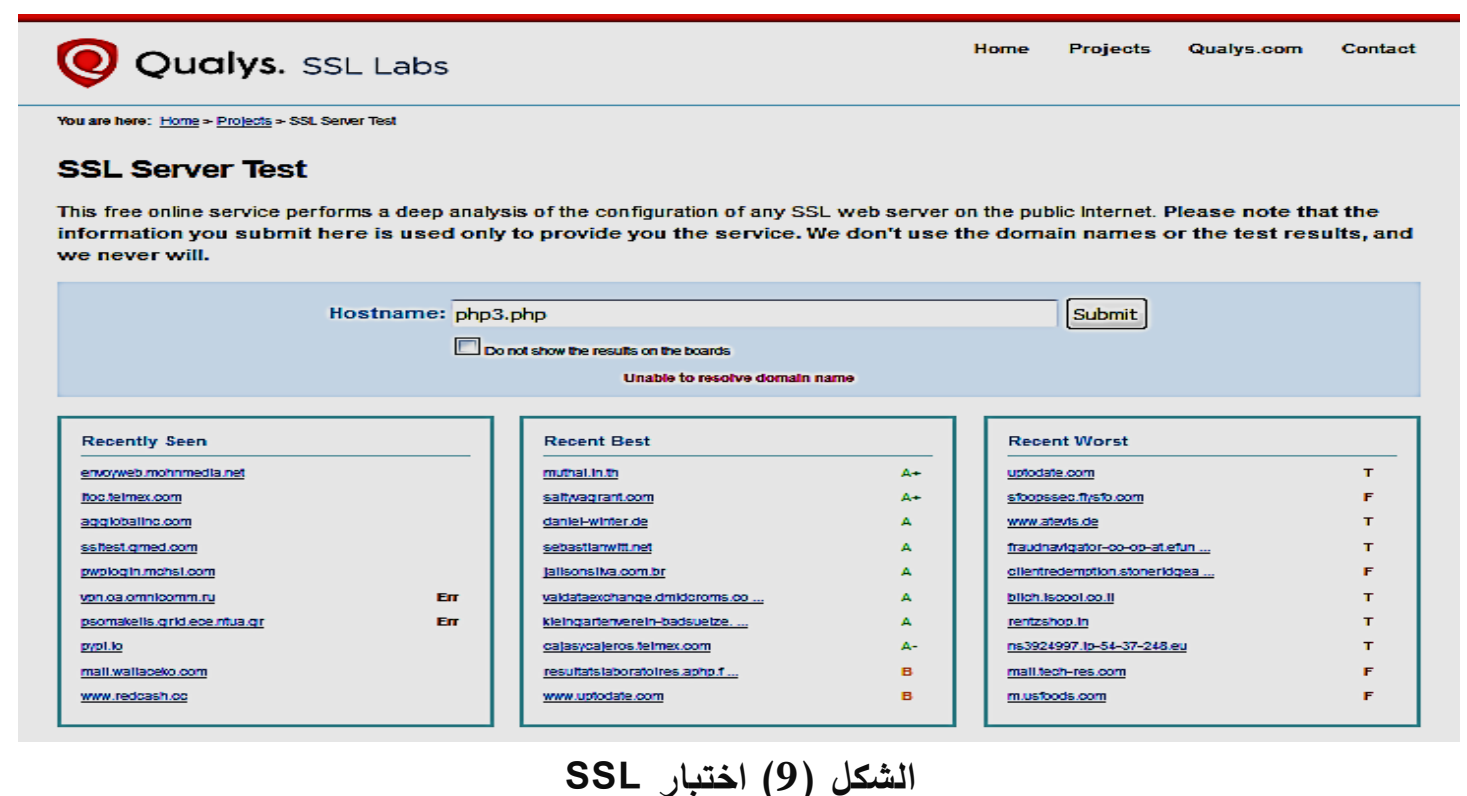

من ملاحظة الثكل(9) يتبين بأن هناك ثلاث مجموعات من مواقع الخدمات المرتبطة ب SSL والتي تقدم خدمات معينة (قوة التثفير , تبديل المفتاح , بروتوكول الدعم , الثهادة , درجة تقييم اداء الخدمة) فالمجموعة الاولى على اليسار تضم كل مواقع الخدمة المرئية

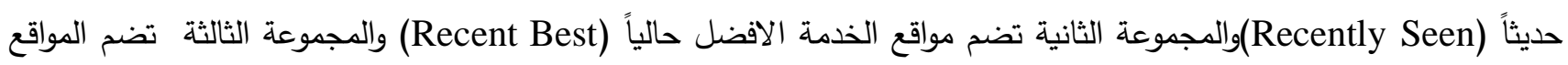
الاسوء خدمة حالياً (Recent Worst). ان كل موقع خدمة من مواقع الخدمة الدذكورة في الثكل (9) تقدم تقييم لأداء الموقع التجاري 
المستخدم وما تقدمه مواقع الخدمة من خدمات حسب تقارير مختبرات SSL .ان الجداول (1) و (6) و(9) توضح تقييم الاداء لكل خدمة مع التقدير • الجداول مقسمة حسب مجموعات مواقع الخدمة الافضل والحالية والاسوء المذكورة في الثكل السابق •

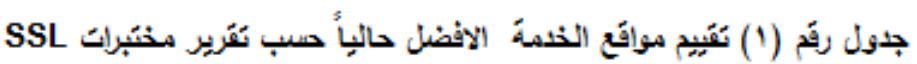

\begin{tabular}{|c|c|c|c|c|c|}
\hline Grade & $\begin{array}{c}\text { Cipher } \\
\text { Strength }\end{array}$ & $\begin{array}{c}\text { Key } \\
\text { Exchange }\end{array}$ & $\begin{array}{l}\text { Protocol } \\
\text { Support }\end{array}$ & Certificate & Name of Service \\
\hline $\mathrm{A}+$ & 90 & 90 & 93 & 100 & muthai.in.th \\
\hline $\mathrm{A}+$ & 90 & 90 & 93 & 100 & saltyvagrant.com \\
\hline A & 90 & 90 & 93 & 100 & daniel-winter.de \\
\hline A- & 90 & 90 & 93 & 100 & cajasycajeros.telmex.com \\
\hline B & 90 & 90 & 97 & 100 & $\begin{array}{l}\text { resultatslaboratoires.aphp.f } \\
\mathbf{r}\end{array}$ \\
\hline
\end{tabular}

يحصل موقع الخدمة على التقدير A+ وهي اعلى نسبة تقدير للأداء اذا كان الموقع يقدم الخدمات بالنسب التالية (قوة الثهادة

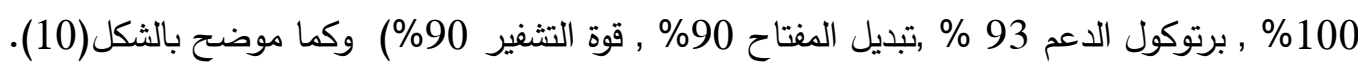

\begin{tabular}{|c|c|}
\hline Summary & \\
\hline Cverall Rating & \\
\hline Cisit our documentation page for more information, configuration guides, and books. Known issues are documented here. \\
\hline This site works only in browsers with SNI support. \\
\hline DNS Certification Authority Authorization (CAA) Policy found for this domain. MORE INFO $\mathrm{n}$ \\
\hline
\end{tabular}

A+ الثكل (10) يوضح نسب الخدمات حسب التقدير

ان خصائص التقدير A+ تكون حسب نسب الخدمات التي يقدمها الموقع اضافة الى تقييم كل خدمة حسب نوعية وقوة الالية المستعملة في الخدمة, بالنسبة للموقع الحاصل على التقدير +A تكون خصائصه موجزة بالجدول (2).

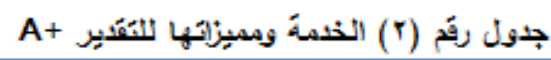

\begin{tabular}{|c|c|}
\hline \multicolumn{1}{|c|}{ Properties } & Service \\
\hline RSA 4096 bits (SHA256withRSA) & Certificate \\
\hline RSA 4096 bits \\
\hline SHA256withRSA & $\begin{array}{c}\text { Signature } \\
\text { algorithm }\end{array}$ \\
\hline $\begin{array}{c}\text { Let's Encrypt Authority X3 } \\
\text { http://cert.int-x3.1etsencrypt.org/ }\end{array}$ & Protocols \\
\hline Handshake, TLS 1.2 TLS 1.1,TLS 1.0 & $\begin{array}{c}\text { Cipher } \\
\text { Suites }\end{array}$ \\
\hline TLS_ECDHE_RSA_WITH_AES_256_GCM_SHA384 & $\begin{array}{c}\text { Handshake } \\
\text { Simulation }\end{array}$ \\
\hline Firefox & TLS_ECDHE_RSA_WITH_AES_128_GCM_SHA256
\end{tabular}

يتم ملاحظة المعلومات في الجدول(2) ان الشهادة مشفرة بأستعمال طريقة RSA لكل 4096 bits مع التوقيع الرقمي, ومفتاح الثفرة

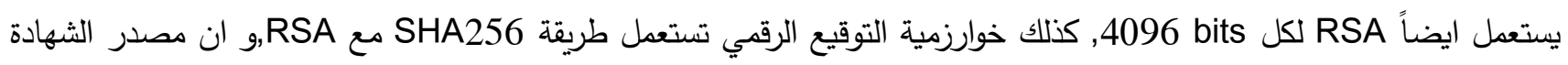

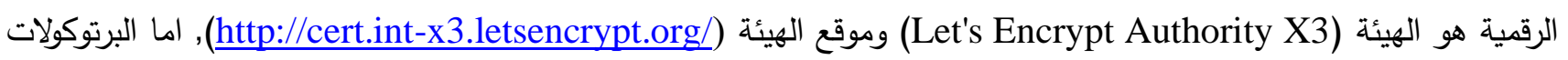


المستعملة (Handshake, TLS 1.2 ,TLS 1.1,TLS 1.0) هنا يتم ملاحظة ان البرتوكول باللون الاخضر هو من الاصدارات الاحدث وملائم جداً مع موقع الخدمة المعني , اما بالنسبة لطرائق التثفير مع البرتوكولات التي يستعطها (AES_TS_ECDHE_RSA Elliptic Curve Diffie- تكون ملائمة . من الجدير بالذكر ان ECDHE (AES_256_GCM_SHA384 هو الاساس لبرتوكول ارتباط الويب الامن SSL التقليدي وهو مدعم بواسطة كل المستعرضات الحديثة] 7]. كذلك تجري مصافحة الموقع مع المتصفح Firefox ( وهو المتصفح المستعمل بالعمل ) عن طريق احدى طرائق التشفير و البرتوكولات المذكورة ( TLS_ECDHE_RSA مع AES_128_GCM _ SH A256).اما اذا كان التقدير A يحصل موقع الخدمة على نسبة عالية للأداء اذا ان الموقع يقدم الخدمات بالنسب التالية ( قوة الشهادة 100\%, برتوكول الدعم93 \% \% قوة التثفير 90\%) وكما مبين بالثكل(11).

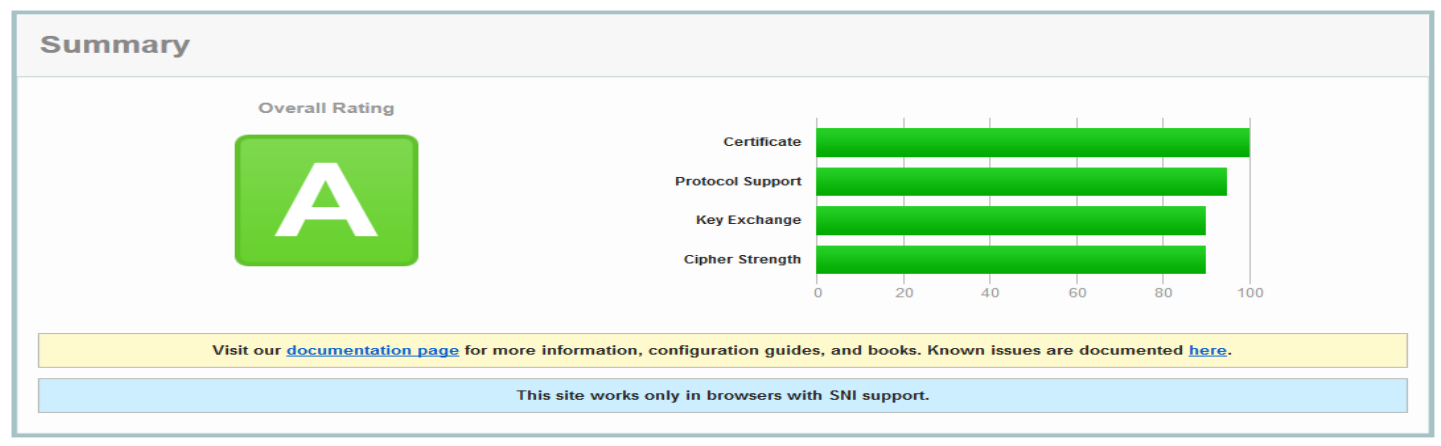

A الشكل (11) يوضح نسب الخدمات حسب التقدير

ان خصائص التقدير A تكون حسب نسب الخدمات التي يقدمها الموقع اضافة الى تقييم كل خدمة حسب نوعية وقوة الالية المستعملة في الخدمة , يجب ان نلاحظ ان مواقع الخدمة هنا تعمل فقط على المتصفحات التي تدعم SNI (هي مختصر Server Name Indication التقدير A تم ايجاز خصائصه في الجدول (3).

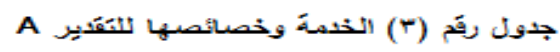

\begin{tabular}{|c|c|}
\hline Properties & Service \\
\hline RSA 2048 bits (SHA256withRSA) & Certificate \\
\hline RSA 2048 bits & Key \\
\hline SHA256withRSA & $\begin{array}{c}\text { Signature } \\
\text { algorithm }\end{array}$ \\
\hline Let's Encrypt Authority X3 & Issuer \\
http://cert_int-x3.1etsencrypt.org/ & Protocols \\
\hline Handshake , TLS 1.2 ,TLS 1.1,TLS 1.0 & $\begin{array}{c}\text { Cipher } \\
\text { Suites }\end{array}$ \\
\hline TLS_ECDHE_RSA_WITH_AES_256_GCM_SHA384 & $\begin{array}{c}\text { Handshake } \\
\text { Simulation }\end{array}$ \\
\hline Firefox
\end{tabular}

من ملاحظة الجدول اعلاه ان الثهادة مشفرة بأستعمال طريقة لكل RSA لكل 2048 مع طريقة التوقيع الرقمي, المفتاح يستعمل

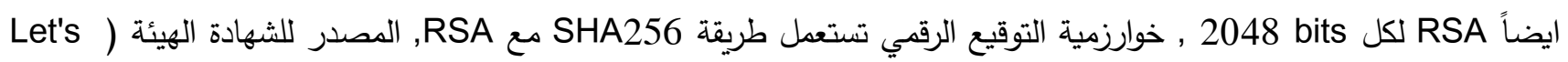
(Encrypt Authority X3 يمكن ملاحظة ان البرتوكول باللون الاخضر هو من الاصدارات الاحدث و 1.0

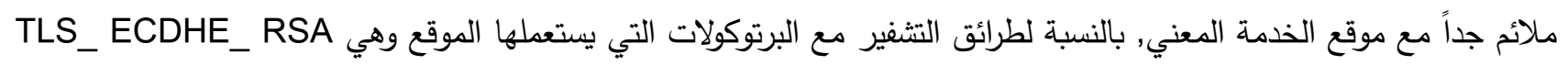
مع Firefox تكون ملائمة , وتجري مصافحة الموقع مع المتصفح ( وهو المتصفح المستعمل

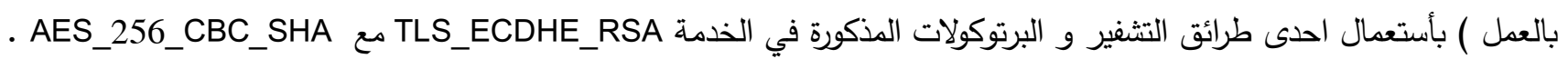


يحصل موقع الخدمة على التقدير - A وهي نسبة تقدير للأداء اذا كان الموقع يقدم الخدمات بالنسب التالية (قوة الثهادة 100\% , برتوكول الدعم 93 \% , تبديل المفتاح 90\% , قوة التثفير 90\%),كما موضح بالثكل (12).

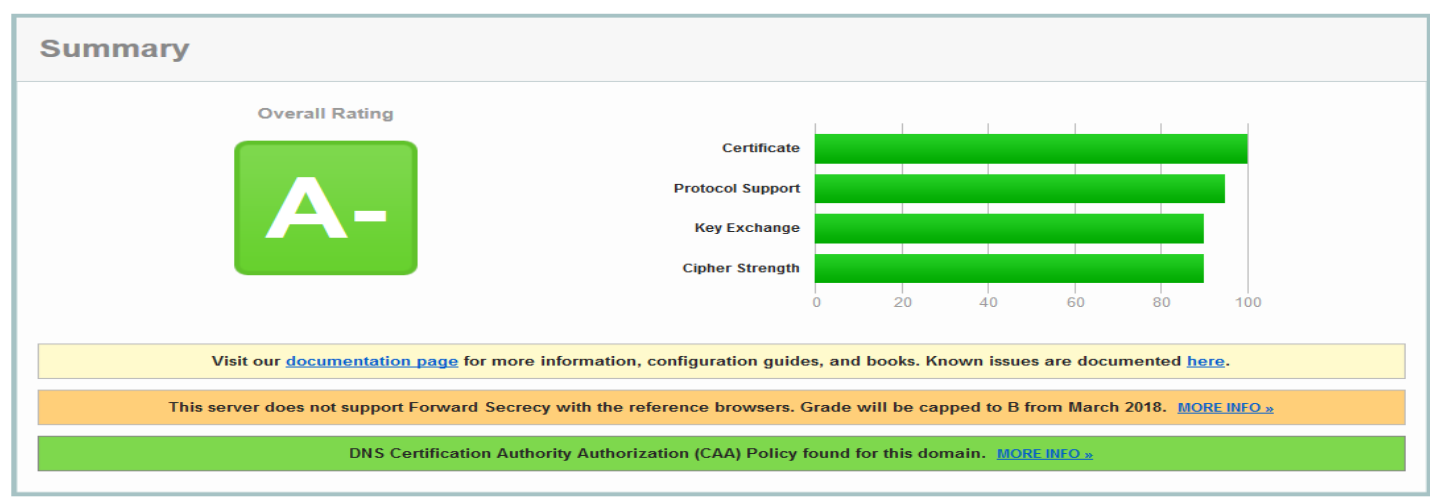

A - الثكل (12) يوضح نسب الخدمات حسب التقدير

ان خصائص التقدير - A تكون حسب نسب الخدمات التي يقدمها الموقع اضافة الى تقييم كل خدمة حسب نوعية وقوة الالية

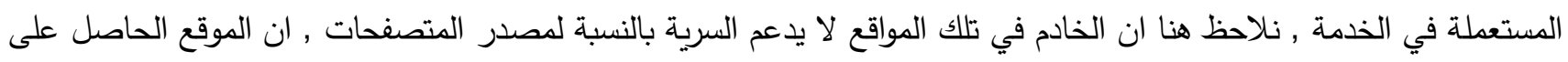
التقدير - A تكون خصائصه موجزه في الجدول (4).

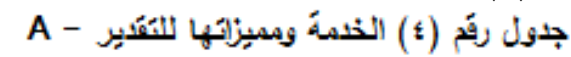

\begin{tabular}{|c|c|}
\hline Properties & Service \\
\hline RSA 2048 bits (SHA256withRSA) & Certificate \\
\hline RSA 2048 bits & Key \\
\hline SHA256withRSA & $\begin{array}{c}\text { Signature } \\
\text { algorithm }\end{array}$ \\
\hline DigiCert SHA2 Secure Server CA & Issuer \\
\hline http://cacerts.digicert.com/DigiCertSHA2SecureServerCA.crt & Protocols \\
\hline Handshake, TLS 1.2 & Cipher Suites \\
\hline Firefox_RSA_WITH_AES_256_GCM_SHA384 WEAK & $\begin{array}{c}\text { Handshake } \\
\text { Simulation }\end{array}$ \\
\hline TLS_RSA_WITH_AES_128_CBC_SHA & \\
\hline
\end{tabular}

من ملاحظة الجدول اعلاه ان الثهادة مشفرة بأستعمال طريقة RSA لكل 2048 bits مع طريقة التوقيع الرقمي, المفتاح

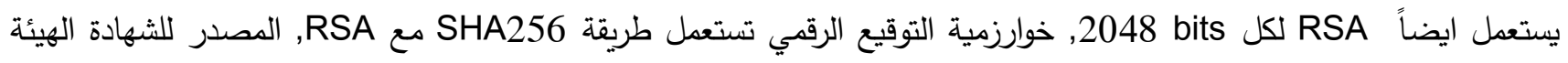
http:// cacerts .digicert .com/ Digi Cert SHA2 ) مع ذكر موقع الهيئة (DigiCert SHA2 Secure Server CA) هecure Server CA.crt

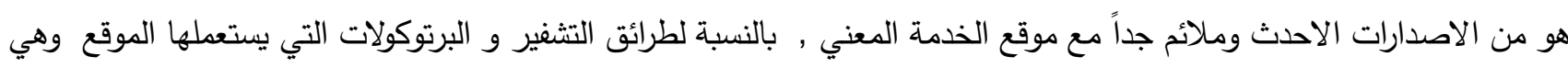

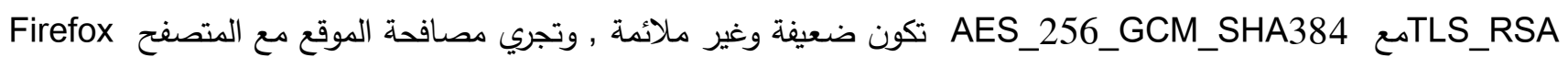

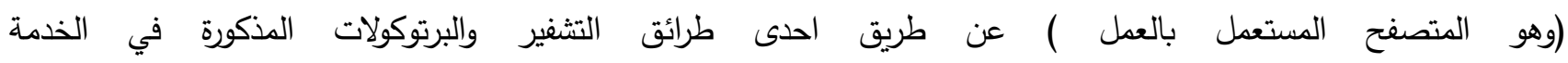
م . AES_128_CBC_SHA مصصل موقع الخدمة على التقدير BTLS_RSA

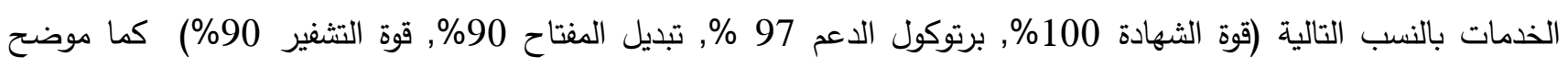
بالثكل(13). 


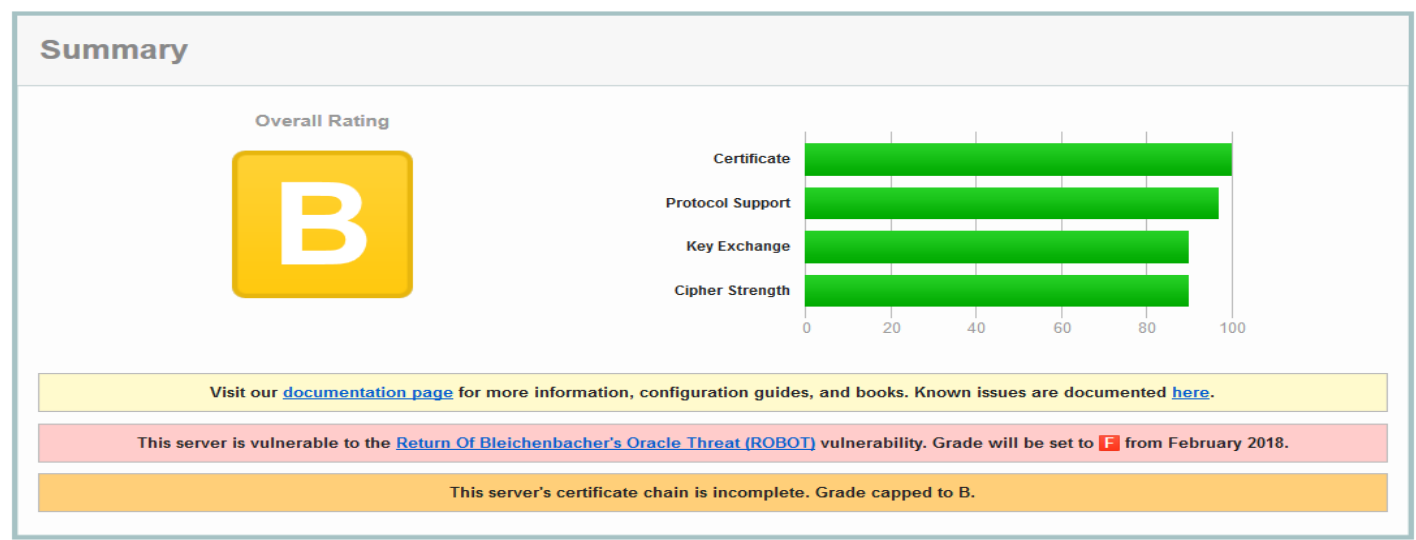

$$
\text { B الثكل (13) يوضح نسب الخدمات حسب التقدير }
$$

ان خصائص التقدير B تكون حسب نسب الخدمات التي يقدمها الموقع اضافة الى تقييم كل خدمة حسب نوعية وقوة الالية المستعملة في الخدمة , نلاحظ هنا ان سلسة الثهادات الرقمية للخوادم غير مكتملة , ان الموقع الحاصل على التقدير B تم ايجاز خصائصه في الجدول (5).

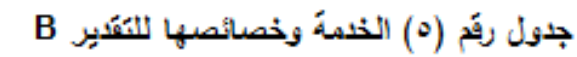

\begin{tabular}{|c|c|}
\hline Properties & Service \\
\hline RSA 2048 bits (SHA256withRSA) & Certificate \\
\hline RSA 2048 bits & Key \\
\hline SHA256withRSA & $\begin{array}{l}\text { Signature } \\
\text { algorithm }\end{array}$ \\
\hline $\begin{array}{c}\text { GlobalSign Domain Validation CA - SHA256-G2 } \\
\text { http://secure.globalsign.com/cacert/gsdomainvalsha2g2r1.crt }\end{array}$ & Issuer \\
\hline Handshake, TLS 1.2, TLS 1.1 & Protocols \\
\hline TLS_ECDHE_RSA_WITH_AES_128_GCM_SHA256 & Cipher Suites \\
\hline $\begin{array}{l}\text { Firefox TLS_ECDHE_RSA_WITH_AES_128_GCM_SHA256_ } \\
\text { ECDH }\end{array}$ & $\begin{array}{l}\text { Handshake } \\
\text { Simulation }\end{array}$ \\
\hline
\end{tabular}

من ملاحظة الجدول اعلاه ان الثهادة مشفرة بأستعمال طريقة RSA لكل 2048 bits مع طريقة التوقيع الرقمي , المفتاح يستعمل

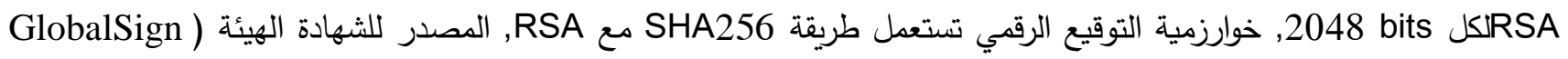
http:// secure. Global) (Domain Validation CA - SHA256 - G2 (البرتوكولات المستعملة ( Handshake, TLS 1.2,TLS 1.1 ) نلاحظ ان موفع (sign.com/cacert/gsdomainvalsha2g2r1.crt البرتوكول باللون الاخضر هو من الاصدارات الاحدث وملائم جداً مع موقع الخدمة المعني , بالنسبة لطرائق التشفير والبرتوكولات التي يستعلها الموقع وهي TLS_ECDHE_RSA مع AES_128_GCM_SHA256 تكون ملائمة , وتجري مصافحة الموقع مع المتصفح Firefox (وهو المتصفح المستعمل بالعمل ) بواسطة طرائق التشفير والبرتوكولات المذكورة في الخدمة

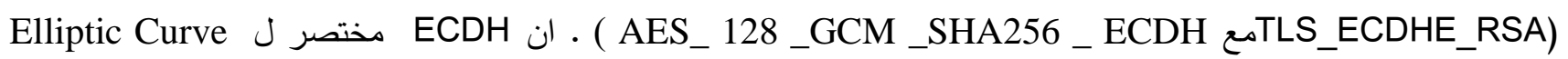

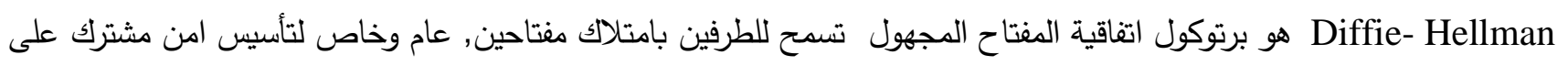

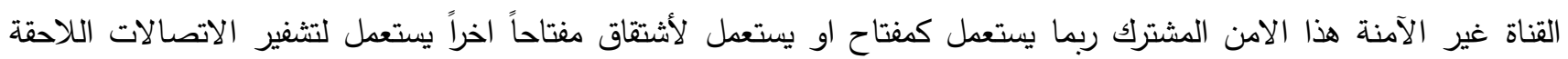

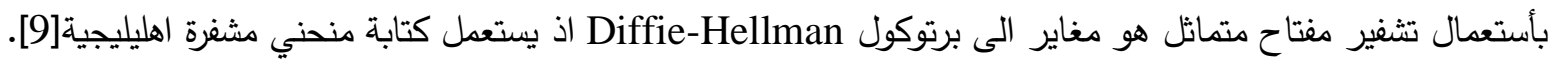




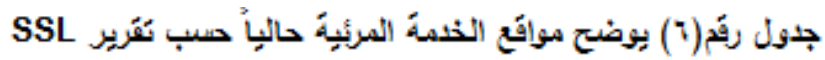

\begin{tabular}{|c|c|c|c|c|c|}
\hline Grade & $\begin{array}{l}\text { Cipher } \\
\text { Strength }\end{array}$ & $\begin{array}{l}\text { Key } \\
\text { Exchange }\end{array}$ & $\begin{array}{l}\text { Protocol } \\
\text { Support }\end{array}$ & Certificate & Name of Service \\
\hline A & 90 & 90 & 93 & 100 & envoyweb.mohnmedia.net \\
\hline A & 90 & 90 & 93 & 100 & itoc.telmex.com \\
\hline A & 90 & 90 & 93 & 100 & mail.wallaceko.com \\
\hline \multicolumn{2}{|l}{ Assessment failed: Unable to connect to the server } & vpn.oa.omnicomm.ru \\
\hline \multicolumn{2}{|l}{ Assessment failed: Unable to connect to the server } & psomakelis.grid.ece.ntua.gr \\
\hline
\end{tabular}

يوضح الجدول رقم (6) مواقع الخدمة المرئية حالياً ان اغلب تقديرات الخدمة هي A وتوجد مواقع غير قادرة على الربط بالخادم حالياً اذ تعطي تقيمات خاطئة . يحصل موقع الخدمة على التقدير F وهي نسبة تقدير للأداء اذا كان الموقع يقدم الخدمات بالنسب التالية (قوة الثهادة 100\% , برتوكول الدعم 50 \% ,تبديل المفتاح 90\% , قوة التثفير 0\%) ويبين الثكل (14) ذلك بوضوح.

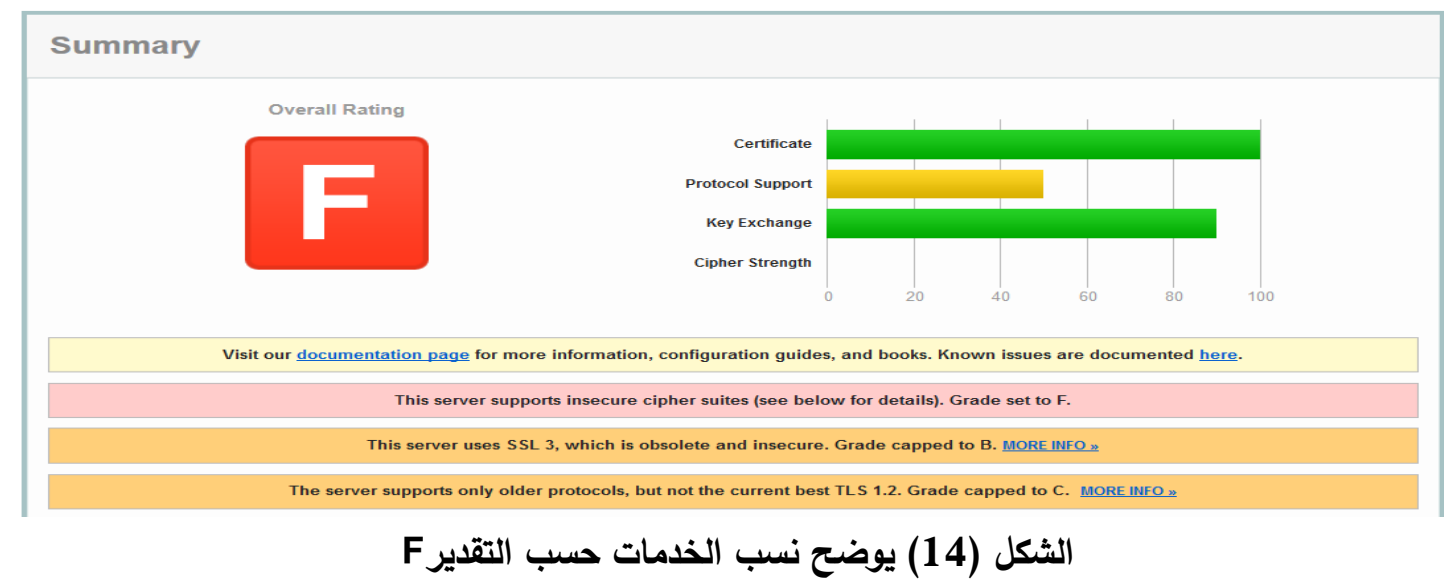

ان خصائص التقدير Fكون حسب نسب الخدمات التي يقدمها الموقع اضافة الى تقييم كل خدمة حسب نوعية وقوة الالية المستعملة

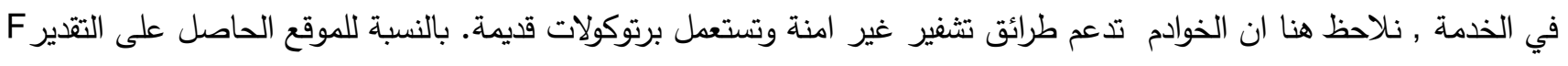
تكون خصائصه موجزه في الجدول (7).

\begin{tabular}{|c|c|}
\hline Properties & Service \\
\hline RSA 2048 bits (SHA256withRSA) & Certificate \\
\hline RSA 2048 bits & Key \\
\hline SHA256withRSA & $\begin{array}{l}\text { Signature } \\
\text { algorithm }\end{array}$ \\
\hline $\begin{array}{l}\text { COMODO RSA Organization Validation Secure Server CA } \\
\text { http://crt.comodoca.com/COMODORSAOrganizationValidationSecureServe } \\
\text { rCA.crt }\end{array}$ & Issuer \\
\hline Handshake, TLS 1.0, SSL 3 INSECURE & Protocols \\
\hline TLS_RSA_WITH_RC4_128_MD5 INSECURE & $\begin{array}{l}\text { Cipher } \\
\text { Suites }\end{array}$ \\
\hline TLS_RSA_WITH_3DES_EDE_CBC_SHA & $\begin{array}{l}\text { Handshake } \\
\text { Simulation }\end{array}$ \\
\hline
\end{tabular}


من ملاحظة الجدول اعلاه ان الثهادة مشفرة بأستعمال طريقة RSA لكل 2048 مع طريقة التوقيع الرقمي , المفتاح

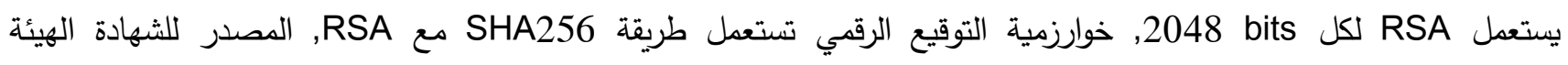
(COMODO RSA Organization Validation Secure Server CA)) http://crt.comodoca.com/COMODORSAOrganizationValidationSecureServerCA.crt المستعملة (Handshake, TLS 1.2,SSL 3 نلاحظ ان البرتوكول باللون الاخضر هو من الاصدارات الاحدث وملائم جداً مع موقع الخدمة المعني بينما SSL 3 المستعمل هنا غير امن , بالنسبة لطرائق التشفير والبرتوكولات التي يستعملها الموقع وهي لاهي

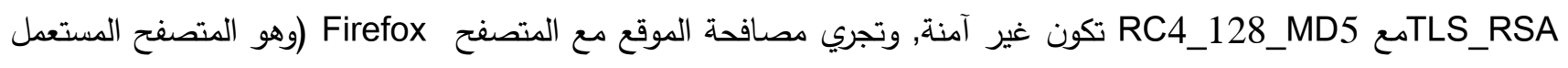

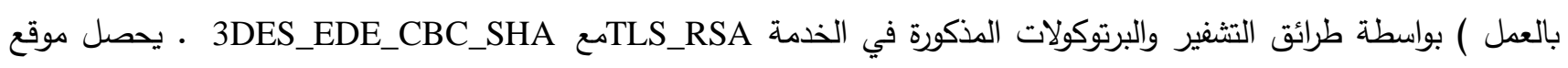

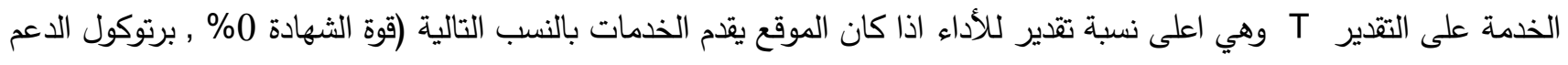
93 \% ,تبديل الففتاح 90\% , قوة التشفير 90\%) وكما مبين بالثكل (15).

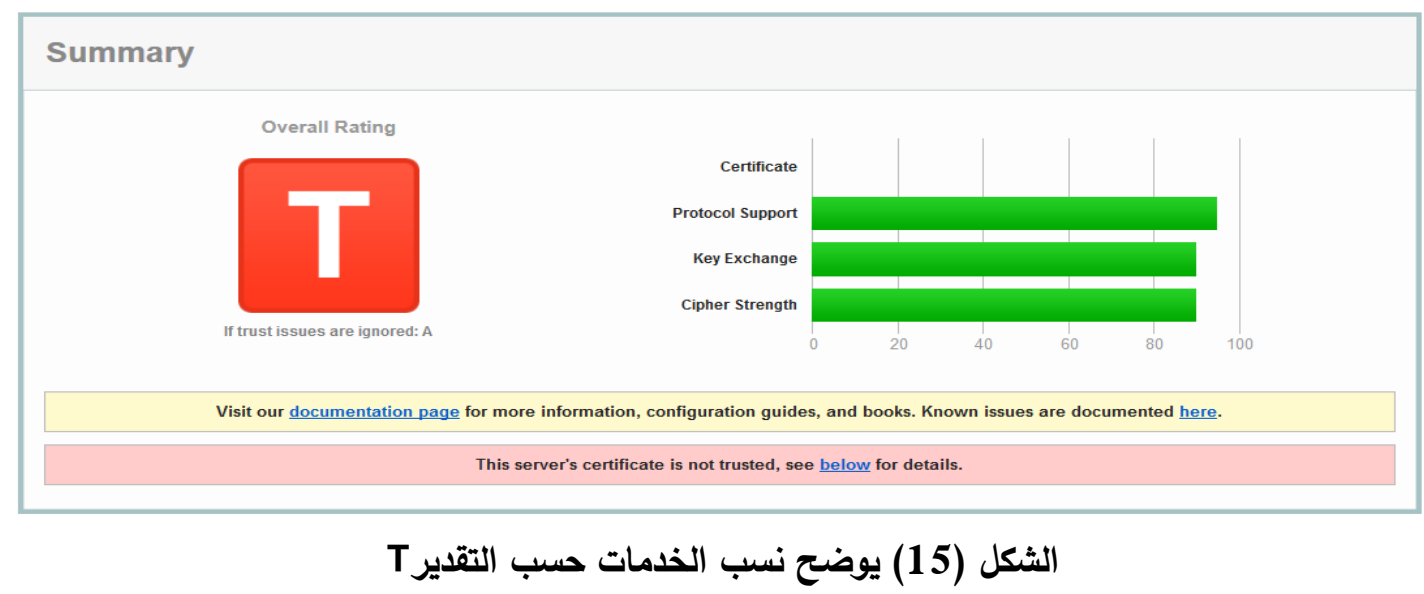

ان خصائص التقدير T تكون حسب نسب الخدمات التي يقدمها الموقع اضافة الى تقييم كل خدمة حسب نوعية وقوة الالية

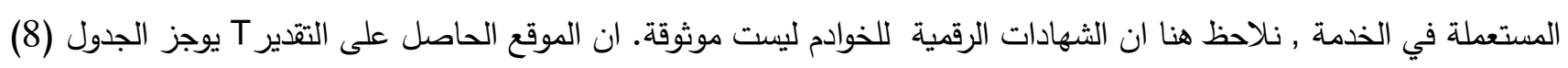
خصائصه.

\begin{tabular}{|c|c|}
\hline Properties & Service \\
\hline RSA 2048 bits (SHA256withRSA), NOT TRUSTED & Certificate \\
\hline RSA 2048 bits & Key \\
\hline SHA256withRSA & $\begin{array}{l}\text { Signature } \\
\text { algorithm }\end{array}$ \\
\hline $\begin{array}{c}\text { COMODO RSA Domain Validation Secure Server CA } \\
\text { http://crt.comodoca.com/COMODORSADomainValidationSecureServerCA. } \\
\text { crt }\end{array}$ & Issuer \\
\hline Handshake, TLS 1.2, TLS 1.1, TLS 1.0 & Protocols \\
\hline TLS_ECDHE_RSA_WITH_AES_256_CBC_SHA384 & $\begin{array}{l}\text { Cipher } \\
\text { Suites }\end{array}$ \\
\hline ECDHE_RSA_WITH_AES_256_CBC_SHA ECDH & $\begin{array}{l}\text { Handshake } \\
\text { Simulation }\end{array}$ \\
\hline
\end{tabular}


من ملاحظة الجدول اعلاه ان الثهادة مشفرة بأستعمال طريقة مSA لكل 2048 bits مع طريقة التوقيع الرقمي لكنها غير

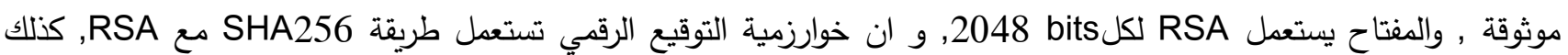

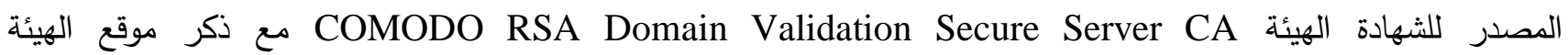
(http://crt.comodoca.com/COMODORSADomainValidationSecureServerCA.crt يمكن ملاحظة ان البرتوكول باللون الاخضر هو من الاصدارات الاحدث وملائم (Handshake, TLS 1.2,TLS 1.1,TLS 1.0 )

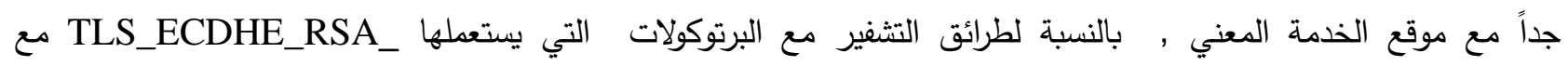

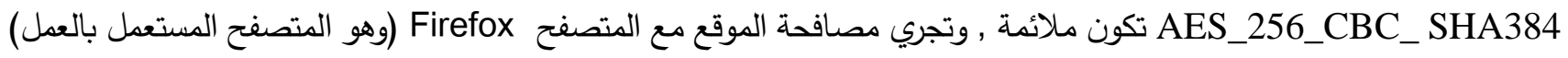

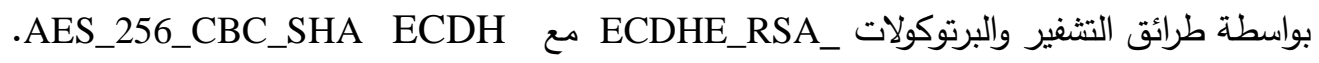
هنالك عدة اسباب لكي تكون الثهادة الرقمية غير موثوقة وقد تمت الاشارة الى المشكلة في التقرير باللون الاحمر , وسببها احدى

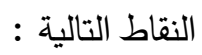

$$
\begin{aligned}
& \text { 1- شهادة رقمية لم يتم التحقق منها } \\
& \text { 2- تهيئة للخادم لم يتم التحقق من صحتها } \\
& \text { 3- الهيئة المانحة للشهادات الرقمية تكون مجهولة }
\end{aligned}
$$

لذلك يجب تطوير البرنوكو لات وطر ائق التشفير من اجل معالجة الاسباب في النقاط 1 و22, اما النقطة الثالثة فيجب التأكد من هيئة

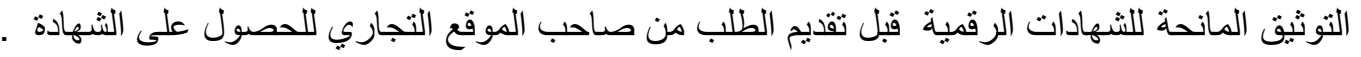

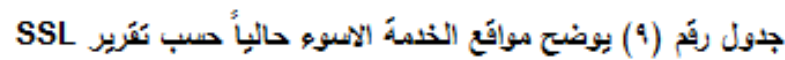

\begin{tabular}{|c|c|c|c|c|c|}
\hline Grade & $\begin{array}{c}\text { Cipher } \\
\text { Strength }\end{array}$ & $\begin{array}{c}\text { Key } \\
\text { Exchange }\end{array}$ & $\begin{array}{c}\text { Protocol } \\
\text { Support }\end{array}$ & Certificate & Name of Service \\
\hline F & 0 & 90 & 50 & 100 & sfoopssec.flysfo.com \\
\hline T & 90 & 90 & 93 & 0 & blich.iscool.co.il \\
\hline T & 90 & 90 & 93 & 0 & rentzshop.in \\
\hline F & 0 & 90 & 50 & 100 & mail.tech-res.com \\
\hline T & 90 & 70 & 93 & 0 & www.atevis.de \\
\hline
\end{tabular}

من ملاحظة الجدول رقم (9) الذي يوضح مواقع الخدمة الاسوء حالياً ان اغلب تقديرات الخدمة هي F و T أي ان مواقع

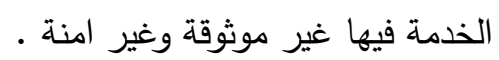
- 4 - الاستنتاج ان من اهم المشاكل التي تواجه التجارة الاككترونية هي التحديات المتعلقة بالامن والخصوصية وحماية المعلومات

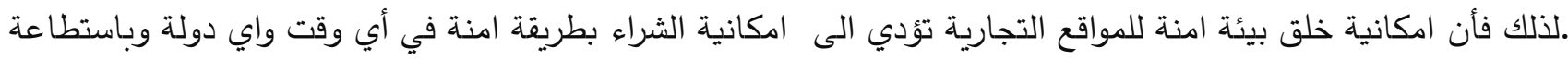

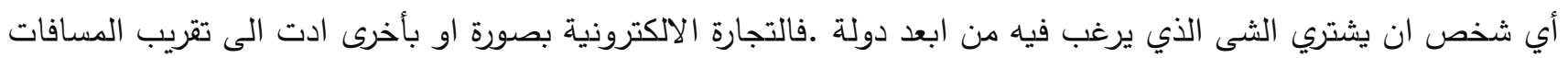
بين الدول. من خلال هذا البحث تم استنتاج ما يلي : لدول

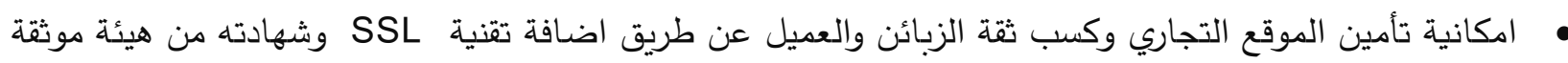

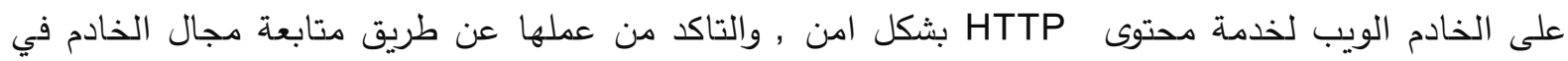
موقع هيئة التوثيق. 


$$
\begin{aligned}
& \text { • العمل على نظام Linux يعطي تأمين وامكانية اعلى مقارنة بنظام Windows , اذ ان الثركات التي تصدر } \\
& \text { الثهادات الرقمية تمنح الخادم ميزات اضافية ممكن برمجتها على نظام Linux لانه مفتوح المصدر , ولام يمكن } \\
& \text { اضافتها في حال استعمال نظام Windows. } \\
& \text { • من خلال درجات التقدير الموجودة في مواقع الخدمة والتي تحدد تقييم الاداء يمكن لصاحب الموقع معرفة درجة }
\end{aligned}
$$

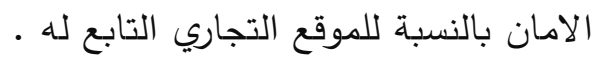

$$
\begin{aligned}
& \text { • من خلال مجال الخادم في موقع هيئة التوثيق ممكن معرفة اذا كانت مواقع الخدمة في حالة صيانة, وبهذا يتوجب التهاب }
\end{aligned}
$$

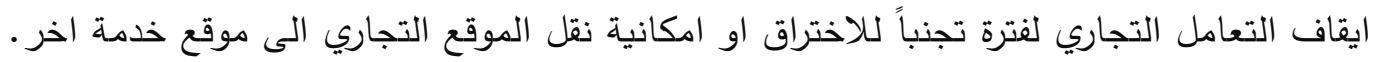

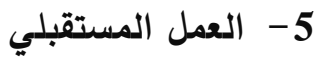

$$
\begin{aligned}
& \text { اضافة تقنية ل SET) Secure Electronic Transactions وهي تقنية تؤمن الصفقات بين الثركات التي تتم عن }
\end{aligned}
$$

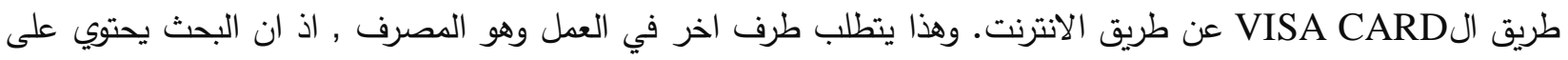

$$
\begin{aligned}
& \text { طرف واحد فقط وهو الموقع التجاري • ويتم اضافة تقنية SET الى خادمي كلا الطرفين و تأمين توقيع ثنائي رقمي لخادم التاجر } \\
& \text { وخادم المصرف . }
\end{aligned}
$$

\section{Acknowledgements:}

Maha Abdul Latif Sayal is a teacher working at Thi-Qar University / College of Computer Science and Mathematics. Bachelor in Computer Science from the College of Science / Thi- Qar University (2005).

Master in Computer Science specialization Cloud Computing College of Science / University of Baghdad (2015), currently PhD student College of Computer Science and Mathematics / University of Kufa.

\section{المصادر}

1- Aba Zaid, d. Thanaa, Tishreen University Journal for Studies and Scientific Research, Economic and Legal Sciences Series Volume 27, No. 4, 2017.

2- Nabil Mahdi Al-Janabi, Muhammad Al-Zaidi, Muhammad Nima, "Economic Intelligence is the only entry point to Knowledge Intelligence", Al-Qadisiyah University, 2018.

3- Ibrahim Ahmed Abdel-Khaleq Al-Dawi, "E-Commerce An Applied Study on Libraries", King Fahd National Library for Printing and Publishing, 2016.

4- Boss, Richard W. "E-Commerce for Libraries". McGraw Hill. August 2007.

5- Thamer Abdul-Ali Kazem Al-Shammari, Fadel Abbas Kazem Al-Shabani, Al-Qadisiyah Journal of Administrative and Economic Sciences, Volume 16, Issue 1, Year 2014. 
6- Kurdish Ahmed El-Sayed, "Arab E-Commerce... Prospects and Challenges," Encyclopedia of Islam and Development, 2017.

7- Hanaa Syed Jawad Al-Nasser, "The Impact of E-Commerce on Competition in Arab Local Markets", Research Study: Arab Democratic Center, 2017.

8- Hala Al-Hassan, Damascus University Journal for Economic and Legal Sciences - Volume 30 - First Issue. 2014

9- William Stallings, "Principles and Practices of Cryptography and Network Security, Fourth Edition," ISBN 10: 0-13-187316-4, Prentice-Hall, 2005.

10- Mohammed Al-Banat, "Electronic Contracts. Symposium on Electronic Commerce Contracts and their Areas", Cairo,Arab Administrative Development Organization, 2013.

11- Farouk Sayed Hussein, "Electronic Commerce and Insurance", Arab Printing House and Hala Publishing and Distribution, 2015. 